\title{
On Increasing the Spectral Efficiency More Than $100 \%$ by User-In-The-Control-Loop
}

\author{
Rainer Schoenen ${ }^{1}$, \\ Communication Networks (ComNets), Faculty 6, RWTH Aachen University, Germany \\ rs@comnets.rwth-aachen.de
}

\begin{abstract}
OFDMA has become the key technology for future cellular wireless networks like the IMT-Advanced systems IEEE 802.16m and 3GPP LTE-A. The advantage of allowing different modulation\&coding schemes (PhyModes) adaptively for each radio resource is at the same time a new disadvantage because the performance is now distance-dependent from the base station (BS) and the total spectral efficiency depends on how user terminals (UTs) are provided with service opportunities. Instead of increasing the effort to support cell-edge users with high data rates this paper investigates the chances of letting the user participate in the process such that his mobility becomes utilitydriven, in a similar way the user behaves in 802.11 hotspot areas. The user's willingness to move to regions of higher SINR must be supported by a display of the current situation (and indications where to move) plus a utility model (lower cost or higher data rate) which motivates moving a distance monotonic in the utility value. By giving input to the user and utilizing the output of his behavior the user becomes a member of the control loop, in a system theoretic sense. The paper shows numeric results of common scenarios and compares the old and new paradigms.
\end{abstract}

Index Terms-IMT-Advanced, LTE, Relays, User-in-the-loop

\section{INTRODUCTION}

$\mathbf{T}$ HE demand for higher data rates is ever increasing. Cellular wireless networks try to keep up with this demand in order to oversize the capacity, so that in busy hours the network is still operational. Technologies to increase the spectral efficiency are highly sophisticated already, so it is unclear if and how an advancement after IMT-Advanced will look like [1]. Multi antenna techniques can multiply the achievable rate, but SU-MIMO only works well in regions of high signal-to-(noise+interference) ratio $(S I N R)$, i.e. when the user terminal (UT) is located in the cell center around a base station (BS). Coordinated transmission schemes can improve the capacity near the cell edge, however at the expense of more radio resources used (e.g., 2-3 BS transmitting) and a signaling overhead and less scheduling freedom of choice, so the outcome is questionable. Multihop techniques (using decode-and-forward relay nodes, $\mathrm{RN}$ ) are reasonable to increase cell edge capacity or coverage [2], [3] but with (low) additional cost and gains typically below $50 \%$ [4].

The near-far dilemma is illustrated in Fig. 11. Due to high pathloss and interference, the offered data rate is one order of magnitude lower at the cell border than close to the base station. Even worse is the situation that the same data rate for a UT occupies almost eight times the amount of resources. This is exactly the ratio between the supported rates [bit/s/Hz] between the highest and the lowest PhyMode (Fig. 2, Table I).

\footnotetext{
${ }^{1}$ Dr. Schoenen is spending 2010 at Carleton University, Canada, collaborating with Dr. Yanikomeroglu
}

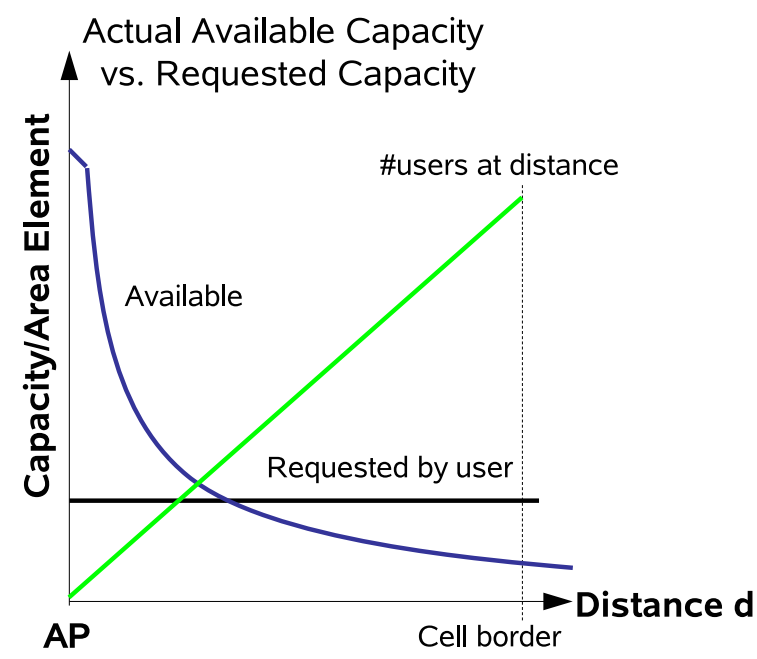

Fig. 1. The near-far problem: With a constant user density the number of users increases with d, so the cell capacity offered per area element differs from the capacity requested by users

In this paper a novel approach is proposed to increase the spectral efficiency. In its uttermost consequence it reflects the user behavior observed very commonly in IEEE $802.11 \mathrm{WiFi}$ hotspot areas. Assuming the UT devices show the current signal quality at the UT position $\overrightarrow{p_{1}}=\left(x_{1}, y_{1}\right)$ (related to the mutual information $M I_{1}$ in bit/s/Hz [5]) and the user has a benefit $b$ of moving towards a location of higher $M I_{2}$ at $\overrightarrow{p_{2}}=\left(x_{2}, y_{2}\right)$, and knows where to go from $\overrightarrow{p_{1}}$ to $\overrightarrow{p_{2}}$, then a certain fraction $p_{M}$ of users will be motivated to do this move.

In a globalized individualistic world the motivation of people to act with reason for the prosperity of the total population is rather limited [6]. But the global challenges demand a rethinking. The current cellular tariff plans (pay per minute, pay per $\mathrm{Kbit} / \mathrm{s}$, flatrate), of which the first two at least lead to reasonable thriftiness, have no element to reflect the different effort (and therefore cost) to support a given data rate. In the present paradigm the network is expected to support the demand anywhere with the same QoS [7]. Therefore the usual cell spectral efficiency is just an average value of all possible $M I(x, y)$, for SISO typically in the interval $[1 ; 2] \mathrm{bit} / \mathrm{s} / \mathrm{Hz}$.

In this paper the previous assumption is canceled. The user becomes involved in the cost process and so the mobility becomes utility-driven. The user is influenced to adjust his location by a noticable incentive for him, so he becomes a part of a closed control loop [8]. Positive user experience and 


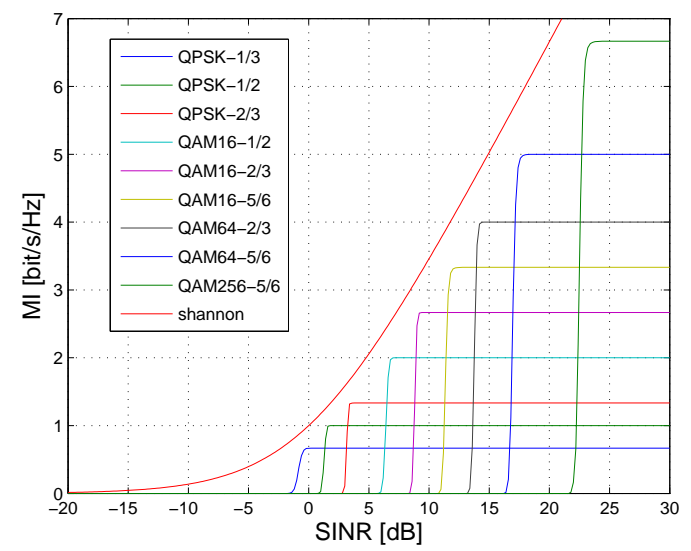

Fig. 2. Link level performance (net MI) for different modulation\&coding schemes (PhyModes). QAM256 is not used here.

not punishment are important for success. Also, an immediate feedback to the user is a psychological advantage. Long delays like not having this until the next phone bill will by far not work as good. Power supply companies recently started to investigate into similar approaches [9].

The initial proposal is to keep tariffs as currently known, but allow savings or payback options on voice calls for those who move to a location of better $M I$, e.g., at certain times of the day (busy hour). Pricing models for traffic become more and more important for engineers anyway [10]. For data traffic the suggestion is a different utility proposal: Let the data rate to/from to a user be proportional to the $M I$, i.e., the scheduler provides a resource fair instead of a rate fair assignment. Not only resource efficiency is an advantage of this approach, but also the green aspect of consuming less energy per bit.

This is one of the popular adcantages of WiFi (802.11) hotspots. The user knows that he can interact and move for improving his performance. This new approach is beneficial for both the user and the operator by means of a better utility (price, rate or QoS) and a higher spectral efficiency.

The paper is organized as follows. The first section defines the utility and mobility models. Then the scenarios of investigation are defined. The last section shows performance results achieved in scenarios based on the IMT-Advanced evaluation [11]. The conclusion summarizes the key contributions.

\section{UTILITY-DRIVEN MOBILITY}

In this paper it is assumed that a UT can observe the current signal quality $M I\left(\overrightarrow{p_{1}}\right)$ at position $\overrightarrow{p_{1}}=\left(x_{1}, y_{1}\right)$. Plus, the user has a tariff model that encourages him to change his position to another location $\overrightarrow{p_{2}}=\left(x_{2}, y_{2}\right)$ if he has a utility advantage of $\Delta u_{1,2}=u\left(\overrightarrow{p_{2}}\right)-u\left(\overrightarrow{p_{1}}\right)$. This utility $u$ can be either financial $(\$ \propto u)$ or an increased data rate. Assume also that the user has all information to make his decision and a suitable UT device (e.g., with GPS ${ }^{1}$ built in), so he knows which direction to move for an improvement and what distance $d_{1,2}$ is required for each improvement step. Figure 3 shows an example how this may look like. The user now

\footnotetext{
${ }^{1}$ without GPS, the network operators can still support ranging by BS-based triangulation and give hints for movement
}

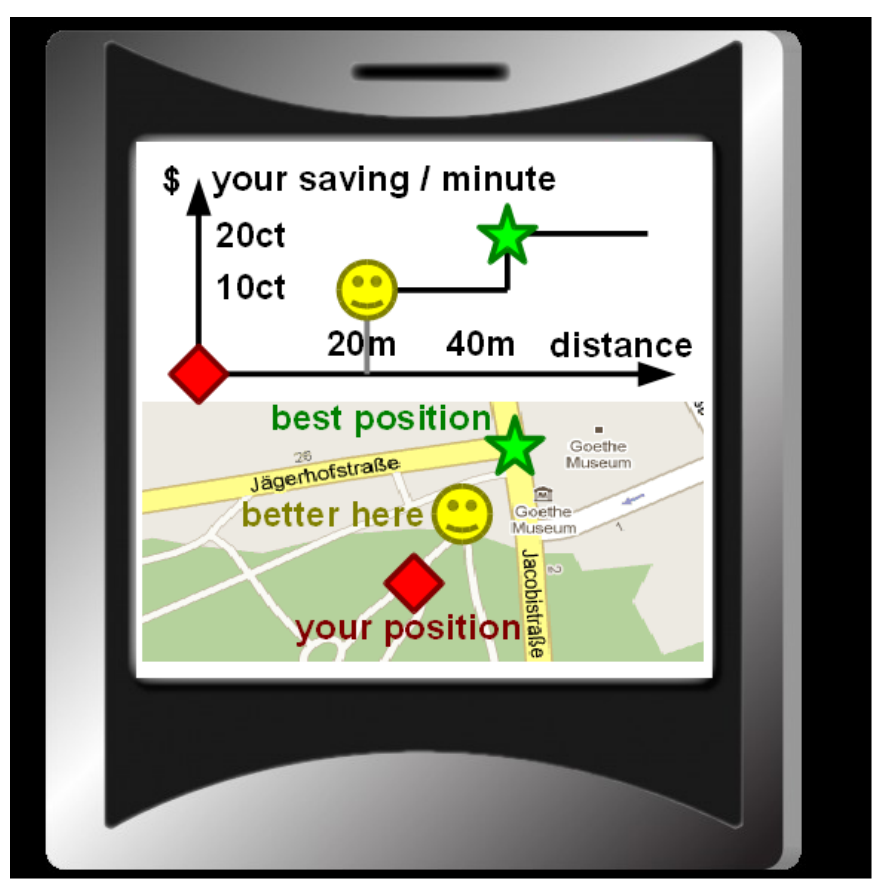

Fig. 3. Example of feedback (input) to user at his UT display. If the benefit for the user is attractive, he will move to a different location with higher spectral efficiency with probability $p_{M}$.

becomes a part of the closed loop system, which is a hybrid of technical and human system blocks. System theory including human elements is not new [12]. Figure 4 shows the system diagram. There is an input to the user block given by $d(m)$ and $u(m)$, meaning the distance and utility for the MI levels $m$ (Table I).

The user itself is expected to decide whether he follows the suggestion or not. There is no coercion to behave according to the proposal, but there must be a motivation to do so. When he is not subscribed to the proposed plan, this is counted as No. The output of the user block is a (movement to a) new location $\overrightarrow{p_{2}}$, which is a random process which can be anywhere between 0 and $d_{1,2}$ meters. For simplicity, but without loss of generality, it is assumed as a Bernoulli random process with $p_{M}$ being the probability of a move to $\overrightarrow{p_{2}}$, where the highest $M I \geq M I_{\text {thresh }}$ is nearby, and $\left(1-p_{M}\right)$ for no movement at all. $M I_{\text {thresh }}$ is the least $M I$ to achieve after the movement (index $m_{\text {thresh }}$ correspondingly). These are expected to be the main parameters describing the user behavior. The motivation aspect itself (from financial or rate benefit to $p_{M}$ ) is not treated in this paper. A more elaborate model would be a probability mass function $M\left(m_{2} \mid m_{1}\right)$ for the probability to go to a location with MI index $m_{2} \geq m_{\text {thresh }}$ when the user is currently at $m_{1}$. Also, in a further step, there can be a 'motivation probability density distribution' $\left.\operatorname{Pr}\left\{d_{1,2} \mid m_{2}, m_{1}\right)\right\}$ which describes the probability of a user to move a distance $d_{1,2}$ to obtain his utility, because there might be less motivation to move more than $100 \mathrm{~m}$. In this paper the first model $p_{M}$ is analyzed and the analysis provides probabilities of average $\bar{d}$ made by the $\left(100 \cdot p_{M}\right) \%$ users who follow the suggestion. 


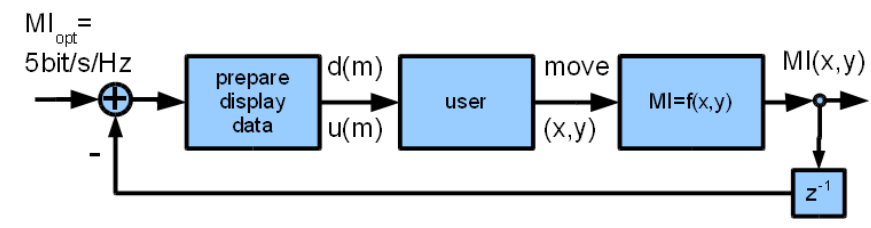

Fig. 4. The user becomes a part of the system (in the loop)

TABLE I

PHYMODES AND SINR INTERVALS

\begin{tabular}{|c|c|c|c|c|c|c|c|c|}
\hline Index $m$ & 1 & 2 & 3 & 4 & 5 & 6 & 7 & 8 \\
\hline SINR & 0.9 & 2.1 & 3.8 & 7.7 & 9.8 & 12.6 & 15.0 & 18.2 \\
\hline Mod. & \multicolumn{5}{|c|}{ QPSK } & \multicolumn{5}{c|}{ QAM16 } & \multicolumn{2}{c|}{ QAM64 } \\
\hline Cod. & $1 / 3$ & $1 / 2$ & $2 / 3$ & $1 / 2$ & $2 / 3$ & $5 / 6$ & $2 / 3$ & $5 / 6$ \\
\hline MI & $2 / 3$ & 1 & $4 / 3$ & 2 & $8 / 3$ & $10 / 3$ & 4 & 5 \\
\hline
\end{tabular}

\section{Performance Models and Scenarios}

For the analysis the IMT-Advanced scenarios were taken as reference [11]. Table III] shows the main parameters and Table II gives the technology parameters according to LTEAdvanced. The analysis takes into account the two-pathloss model (LOS,NLOS) with probability $p_{L O S}[13],[14]$ and calculates the steps given in the sequence below. For the intentional user movement it is assumed that the LOS properties hold at the destination location $\overrightarrow{p_{2}}$, with a linear increase of $p_{L O S}$ to 1.0 from distance $0 \mathrm{~m}$ to $d_{L O S}$ (here $d_{L O S}=10 \mathrm{~m}$ assumed).

- Transmit Power $P_{T x}$ : see Table III,

- Pathloss: see Table [II] and [11] [14],

- Interference I: neighbor cell BSs and neighbor sectors interfere ( $100 \%$ load, cluster order 1)

- Noise $N$ : accounted for but not serious ( $I$-limited),

- SINR: $S I N R=S /(N+I)$,

- MI: mutual information $M I=f(S I N R, \bmod )$ [15],

- BER: bit error ratio, depends on $M I$,

- PER: packet error ratio, the result after channel decoding,

- Throughput: determined by bandwidth, PhyMode (modulation and code rate), ARQ overhead,

- Cell Spectral Efficiency: net spectral efficiency MI [bit/s/Hz] is throughput per bandwidth averaged over the cell (sector) area [15],

- Relays: least resources $\mathrm{BS} / \mathrm{RN}$ association [15],

In addition to the cellular layout with neighbor interference as in the model above, a particular realistic city scenario [16] (shown in Figure 5, with 13 relays) has also been investigated. It will be referred to as the Jersey scenario. Here we can see the effects of shadowing and how easy it is for a user to move to a point of better coverage. The initial user density is assumed constant in the area bounded by the green polygon. The population density after movement is expected to be higher close to the streets and in the city center.

\section{Numerical Results}

Numeric results based on analysis have been obtained. The cell spectral efficiency is the calculated $M I(x, y)$, averaged

\footnotetext{
${ }^{2}$ the decision of single or multihop (relayed) route is taken by considering which option uses less resources, not by $\max (S I N R)$
}

TABLE II

TECHNOLOGY PARAMETERS ACCORDING TO LTE-A

\begin{tabular}{|c|c|}
\hline Bandwidth [MHz] & FDD: 20DL,20UL \\
\hline Traffic & full load; best effort \\
\hline Antenna gain (boresight) & $17 d B i$ \\
\hline Antenna aperture horizontal $\theta_{3 d B}$ & $70^{\circ}$ \\
\hline Antenna aperture vertical $\phi_{3 d B}$ & $15^{\circ}$ \\
\hline Thermal noise & $-174 d \mathrm{dm} / \mathrm{Hz}$ \\
\hline UT noise figure & $5 \mathrm{~dB}$ \\
\hline
\end{tabular}

TABLE III

IMT-ADVANCED SCENARIO SPECIFICATIONS

\begin{tabular}{|c|c|c|c|c|}
\hline Scenario & $\begin{array}{c}\text { Urban } \\
\text { micro } \\
\text { UMi }\end{array}$ & $\begin{array}{c}\text { Urban } \\
\text { macro } \\
\text { UMa }\end{array}$ & $\begin{array}{c}\text { Suburban } \\
\text { macro } \\
\text { SMa }\end{array}$ & $\begin{array}{c}\text { Rural } \\
\text { macro } \\
\text { RMa }\end{array}$ \\
\hline$d_{B S-B S}$ & $200 \mathrm{~m}$ & $500 \mathrm{~m}$ & $1299 \mathrm{~m}$ & $1732 \mathrm{~m}$ \\
\hline$h_{B S}$ & $10 \mathrm{~m}$ & $25 \mathrm{~m}$ & $35 \mathrm{~m}$ & $35 \mathrm{~m}$ \\
\hline$r_{\min }$ & $10 \mathrm{~m}$ & $25 \mathrm{~m}$ & $35 \mathrm{~m}$ & $35 \mathrm{~m}$ \\
\hline Ant. tilt $\phi_{t}$ & $-12^{\circ}$ & $-12^{\circ}$ & $-6^{\circ}$ & $-6^{\circ}$ \\
\hline$f_{C}[G H z]$ & 2.5 & 2.0 & 2.0 & 0.8 \\
\hline$P_{T x}$ & $44 \mathrm{dBm}$ & $49 \mathrm{dBm}$ & $49 \mathrm{dBm}$ & $49 \mathrm{dBm}$ \\
\hline
\end{tabular}

over all points $(x, y)$ of the cell area. The user movement is modeled by circularly searching for each point $\overrightarrow{p_{1}}$ the nearest point $\overrightarrow{p_{2}}$ where $M I\left(\overrightarrow{p_{2}}\right) \geq M I_{\text {thresh }}$ and assuming this $M I\left(\overrightarrow{p_{2}}\right)$ as the new $M I^{\prime}\left(\overrightarrow{p_{1}}\right)$. At the same point the distance $d_{1,2}$ is recorded. Both increases of $\Delta M I$ and $d$ are weighted with $p_{M}$, because $\left(1-p_{M}\right)$ of the users are not willing to move. Users who don't need to move because they're already at a good position account with $d=0$. In the following, HUD means homogeneous user distribution (conservative model) and UIL means user-in-the-loop (progressive model) with anisotropic user density.

Table IV shows the spectral efficiency and distance results for the IMT-Advanced scenarios defined in table III] and [11] (HUD part from [14]). For these results rather moderate values for the parameters have been chosen: $p_{M}=\frac{1}{2}$ and $M I_{\text {thresh }}=2.5 \mathrm{bit} / \mathrm{s} / \mathrm{Hz}$. With an effective movement

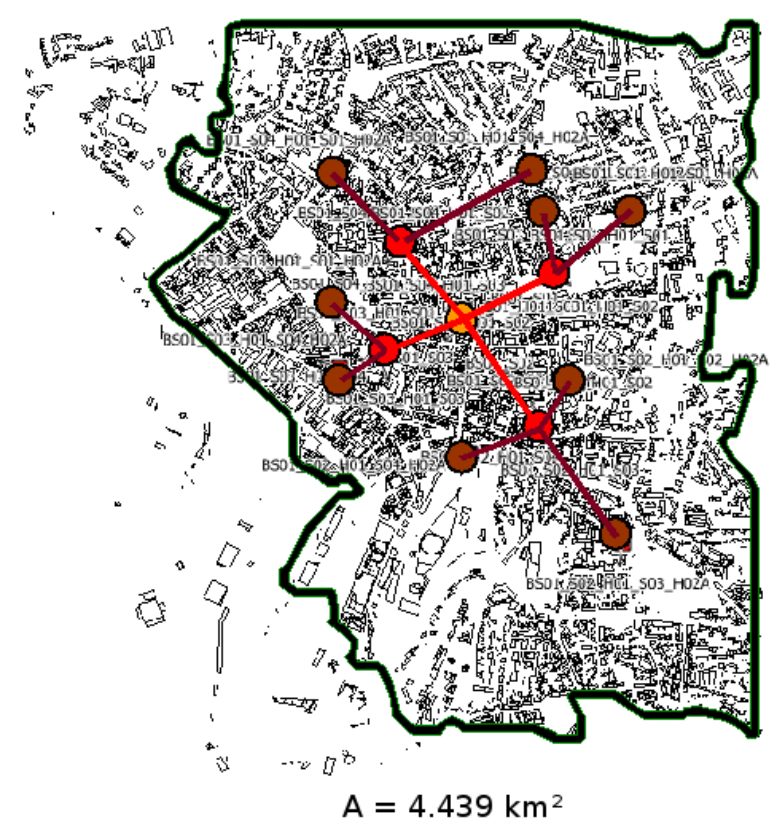

Fig. 5. Scenario map of Jersey [16]: One BS (middle) and a hierarchical $\mathrm{RN}$ placement as well as the polygon of interest (green) 
TABLE IV

GROSS SPECTRAL EFFICIENCY RESULTS FOR THE IMT SCENARIO EVALUATION [BIT/S/Hz/SECTOR] WITH $p_{M}=\frac{1}{2}$ AND $M I_{\text {thresh }}=2.5 \mathrm{bit} / \mathrm{s} / \mathrm{Hz}$

\begin{tabular}{|c|c|c|c|c|}
\hline Scenario & UMi & UMa & SMa & RMa \\
\hline 3S,0RN,HUD & 1.567 & 1.254 & 1.234 & 1.974 \\
\hline 3S,0RN,UIL & 2.170 & 1.995 & 2.836 & 2.509 \\
$\bar{d}=$ & $4.4 \mathrm{~m}$ & $4.7 \mathrm{~m}$ & $7.8 \mathrm{~m}$ & $30.7 \mathrm{~m}$ \\
\hline 3S,3RN,HUD & 1.945 & 1.804 & 1.825 & 2.310 \\
\hline 3S,3RN,UIL & 2.333 & 2.239 & 2.858 & 2.654 \\
$\bar{d}=$ & $1.9 \mathrm{~m}$ & $1.7 \mathrm{~m}$ & $5.0 \mathrm{~m}$ & $12.0 \mathrm{~m}$ \\
\hline
\end{tabular}

TABLE V

GROSS SPECTRAL EFFICIENCY RESULTS FOR THE JERSEY SCENARIO EVALUATION $[$ bit $/ \mathrm{s} / \mathrm{Hz} /$ Sector $]$ AND AVERAGE MOBILITY $\bar{d} /[\mathrm{m}]$

\begin{tabular}{|c|c|c|c|}
\hline Scenario & SpecEff & coverage & $d$ \\
\hline Jersey,0RN,HUD & 1.516 & $69.9 \%$ & $0 \mathrm{~m}$ \\
\hline Jersey,0RN,UIL & 2.350 & $(100 \%)$ & $11.9 \mathrm{~m}$ \\
\hline Jersey,13RN,HUD & 2.358 & $99,1 \%$ & $0 \mathrm{~m}$ \\
\hline Jersey,13RN,UIL & 2.771 & $(100 \%)$ & $11.9 \mathrm{~m}$ \\
\hline
\end{tabular}

of just a few meters the total spectral efficiency could be increased by $25 \%$ to more than $100 \%$, depending on the IMT scenario. It can also be observed that relays reduce the effort for the user to move, because a RN might be closer to his position. Figure 7 shows from where the users need to move and how far, if they want to achieve the benefit.

Figure 10 and Table $\mathrm{V}$ show the results over the cell area of the Jersey scenario of Figure 5 (HUD part see [16]). A significant improvement can be achieved while the average movement is quite "convenient" for the user.

Next the influence of the parameters $p_{M}$ and $M I_{\text {thresh }}$ is studied. We have a look at the total cell spectral efficiency $M I$ and the distance of movement $\bar{d}$. Figure 8 shows the increase of spectral efficiency we can really achieve by user-in-thecontrol-loop. As we can see, a factor of 3 or an increase of $200 \%$ is possible and even a moderate gain is easy to achieve with just a minority of people involved. Obviously the dependency on $p_{M}$ is linear, which appears natural given how it is incorporated. $M I_{\text {thresh }}$ has a nonlinear (piecewise linear) influence, simply because of the switching points of Table $\mathbb{I}$ In Figure 9 we observe that the distance $\bar{d}$ is not so high, taken absolutely. With less $M I_{\text {thresh }}$ it is more convenient for the user to find the next best location but at the same time the total spectral efficiency is less compared to, e.g., moving to a place where $M I(x, y)=5 b i t / s / H z$.

Comparing 0 and 3 relays in Figure 9 we observe only a difference where $M I_{\text {thresh }} \leq 2.5 \mathrm{bit} / \mathrm{s} / \mathrm{Hz}$. This is reasonable, because a two-hop transmission can never achieve more than half of the capacity of the highest PhyMode in Table [1. If this is acceptable $\left(M I_{\text {thresh }}\right)$, the user saves effort $(d)$; otherwise the relay cannot be taken by those users. In the end, when more users cooperate (higher $p_{M}$ ), the highest spectral efficiency is not only a theoretical goal.

\section{CONCLUSION}

This paper presents a new paradigm to let the user actively participate in the process of optimizing the resource usage. For him this is motivated by a utility, either financial or higher data rate. This cancels the conservative paradigm of constant user density and equal service provisioning regardless of different costs. The method was then applied to the IMTAdvanced evaluation scenarios and a realistic city scenario to find out the gain in spectral efficiency and the typical effort required for a user. The obtained results show huge gains up to $200 \%$ without any effort in the physical or MAC layer. Just a slow location-dependent database and a GUI application is required. Also the distances to move are easy to reach on foot. For the motivation, a promising tariff contract or a resourcefair data rate assignment is advised. Future work will take more elaborate user statistics into account. Also the financial aspect of tariff income and reduced infrastructure investment costs can be studied.

The author recommends not to promise the customers ubiquitous equal service quality anymore. Instead, announce that it is location dependent and let him contribute to the common benefit. In the future this may even be extended to the time domain (not only space), in order to reduce the load at busy hours and to improve the overprovisioning (in)efficiency.

\section{REFERENCES}

[1] J. Monserrat, P. Sroka, G. Auer, J. Cabrejas, D. Martin, A. Mihovska, R. Rossi, A. Saul, and R. Schoenen, "Advanced Radio Resource Management for IMT-Advanced in WINNER+ (II)," in Proceedings of the ICT-MobileSummit 2010, Florence, Italy, Jun 2010.

[2] R. Pabst, B. Walke, D. C. Schultz, H. Yanikomeroglu, and et al, "RelayBased Deployment Concepts for Wireless and Mobile Broadband Radio," IEEE Communications Magazine, pp. 80-89, Sep 2004.

[3] M. Salem, A. Adinoyi, M. Rahman, H. Yanikomeroglu, D. Falconer, Y.-D. Kim, E. Kim, and Y.-C. Cheong, "An overview of radio resource management in relay-enhanced OFDMA-based networks," IEEE Communications Surveys and Tutorials, vol. 12, no. 3, Q3 2010.

[4] R. Schoenen, W. Zirwas, and B. Walke, "Capacity and Coverage Analysis of a 3GPP-LTE Deployment Scenario," in Proceedings of the BWAW'08 colocated with ICC'08, Beijing, China, May 2008.

[5] K. Brueninghaus and D. e. a. Astely, "Link performance models for system level simulations of broadband radio access systems," in Proceedings of the 17th PIMRC'2005, Sep 2005, pp. 2306-2311.

[6] G. Hardin, "The tragedy of the commons," Science, vol. 20, pp. 124347, 1968.

[7] J. Sanjay and H. Mahbub, Engineering Internet QoS. Artech House, 2002, ISBN 1580533418.

[8] C. Kilian, Modern Control Technology. ISBN 1-4018-5806-6: Thompson Delmar Learning, 2005.

[9] A. Faruqui and R. Earle, "Demand response and advanced metering," Regulation, vol. 29, no. 1, pp. 24-27, 2006.

[10] S. Dixit, S. Periyalwar, and H. Yanikomeroglu, "A distributed framework with a novel pricing model for enabling dynamic spectrum access for secondary users," in Proceedings of the IEEE Vehicular Technology Conference, Sep 2009, p. 6.

[11] ITU, "Report ITU-R M2135; Guidelines for evaluation of radio interference technologies for IMT-Adcanced", 2008.

[12] R. Zimmerman, Das System Mensch. Artech House, 2003, ISBN 300-012784-4.

[13] D. Bültmann, T. Andre, and R. Schoenen, "Analysis of 3GPP LTEAdvanced Cell Spectral Efficiency," in Proc. of the PIMRC'2010, Istanbul, Turkey, September 2010.

[14] R. Schoenen and C. Teijeiro, "System Level Performance Evaluation of LTE with MIMO and Relays in Reuse-1 IMT-Advanced Scenarios," in Proceedings of the IEEE WiCom, Chengdu, China, Sep 2010.

[15] R. Schoenen and B. Walke, "On PHY and MAC performance of 3GLTE in a multi-hop cellular environment," in Proceedings of the IEEE WiCom'2007, Shanghai, China, Sep 2007.

[16] R. Schoenen, W. Zirwas, and B. Walke, "Raising Coverage and Capacity using Fixed Relays in a Realistic Scenario," in Proceedings of the European Wireless Conference, Prague, Jun 2008. 


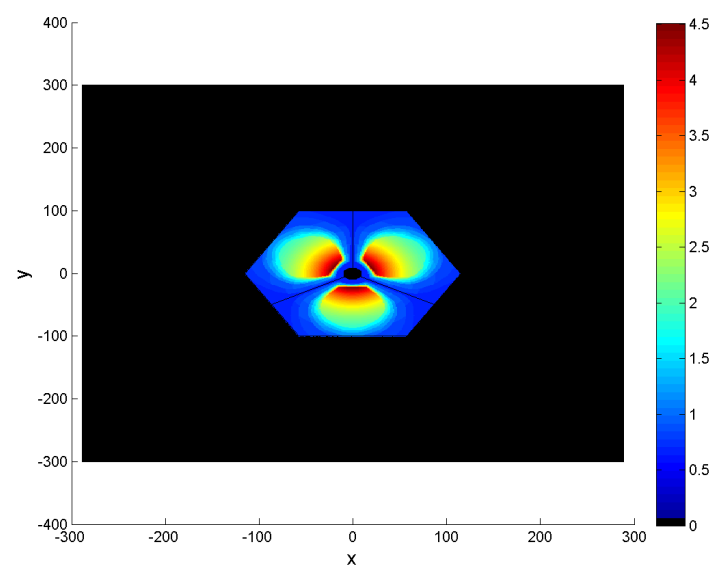

(a) $M I$ of IMT-A scenario UMi

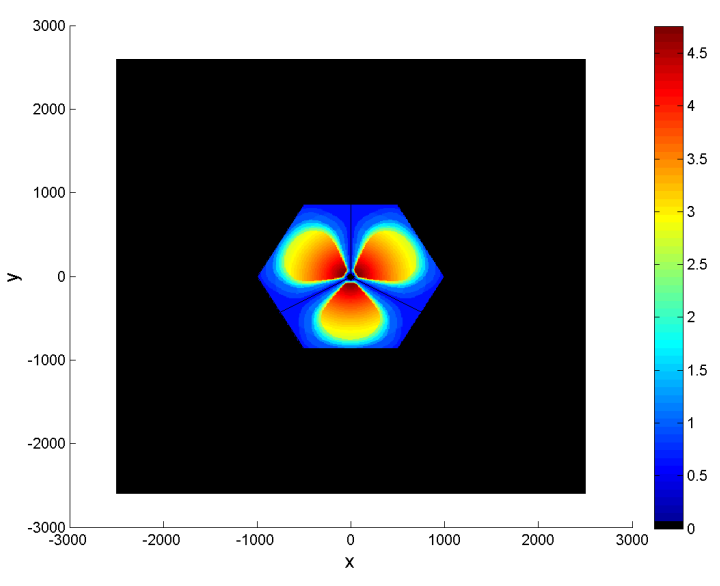

(b) $M I$ of IMT-A scenario RMa

Fig. 6. Example Scenarios (RMa,UMi) of the IMT-Advanced evaluation without our proposed method. The color indicates the $M I$ [bit/s/Hz].

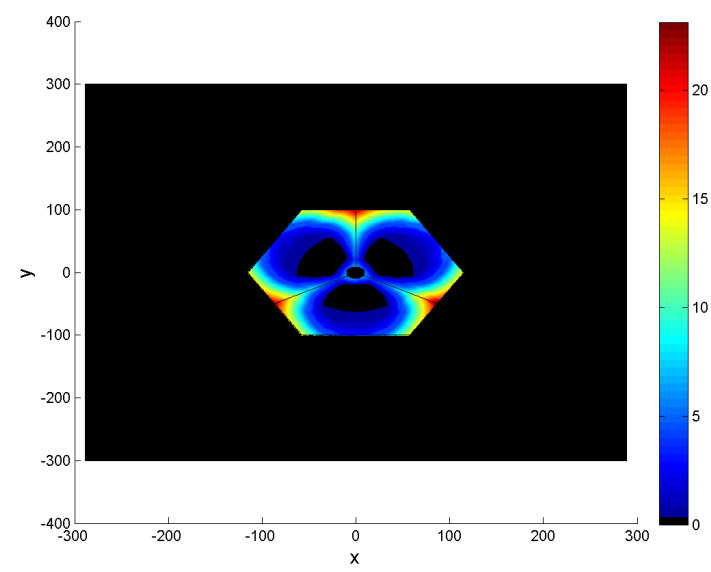

(a) UMi scenario

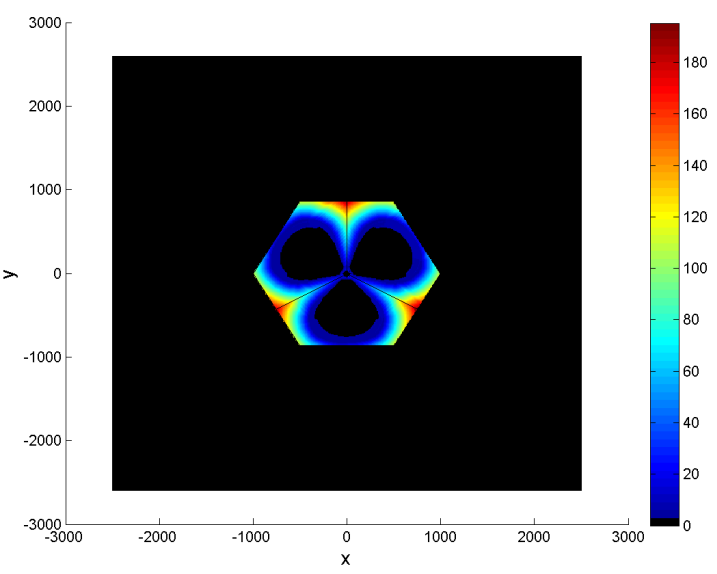

(b) RMa scenario

Fig. 7. Distance of movement $\bar{d} /[m]$ (indicated by color) to achieve best $M I$ with 0 relays, $p_{M}=\frac{1}{2}$ and $M I_{\text {thresh }}=2.5$ bit/s/Hz. In most cases(UMi), a move of the UE of about $5 \mathrm{~m}$ will already result in achieving the best MI

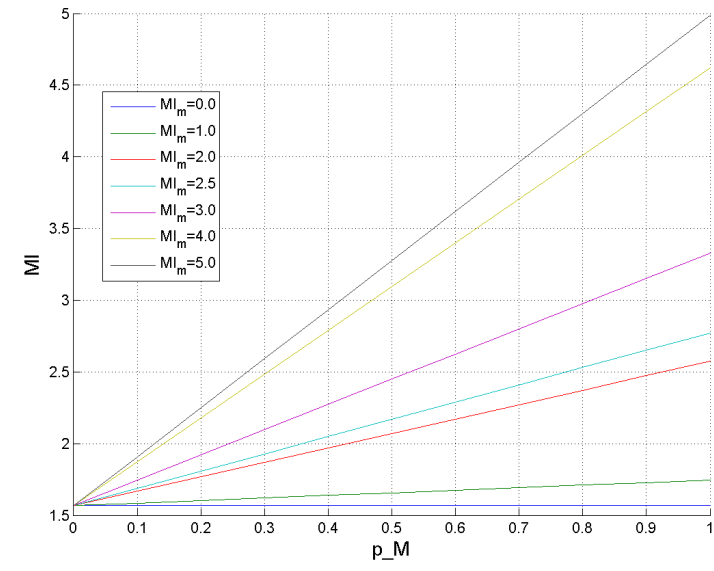

(a) Dependency on $p_{M}$

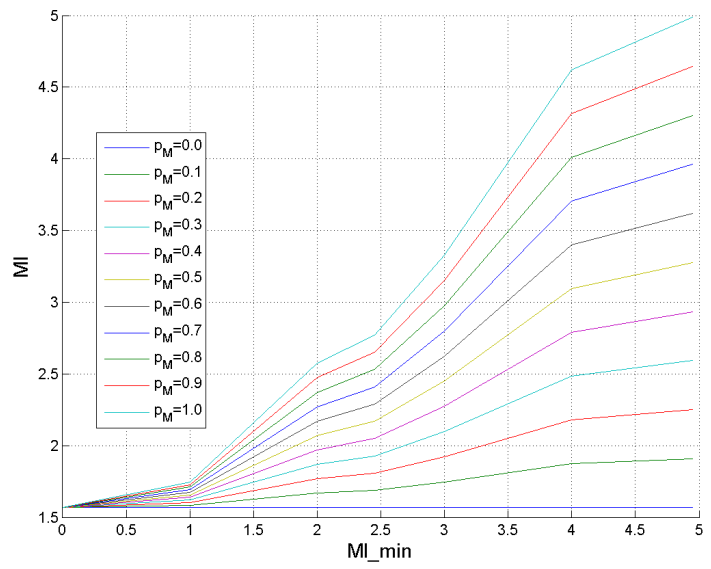

(b) Dependency on $M I_{\text {thresh }}$

Fig. 8. Observed Spectral Efficiency $\bar{M} I /[b i t / s / H z]$ with given parameters in UMi scenario. Obviously a factor of more than three can be achieved. 


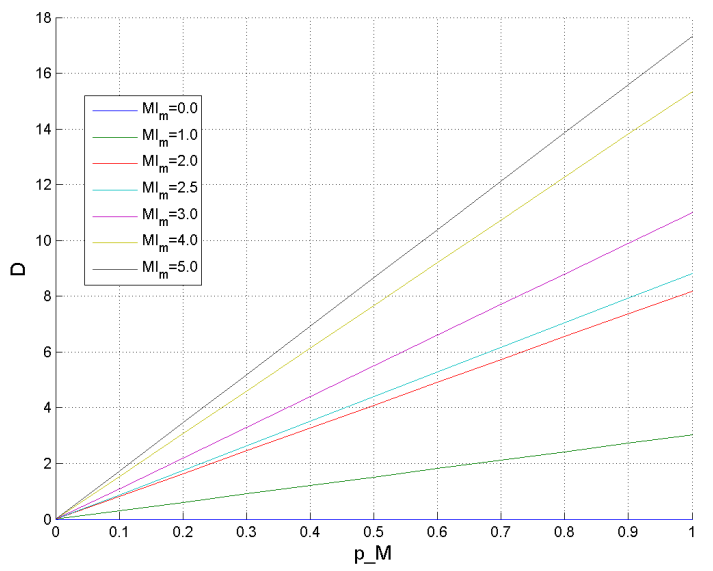

(a) Dependency on $p_{M}$

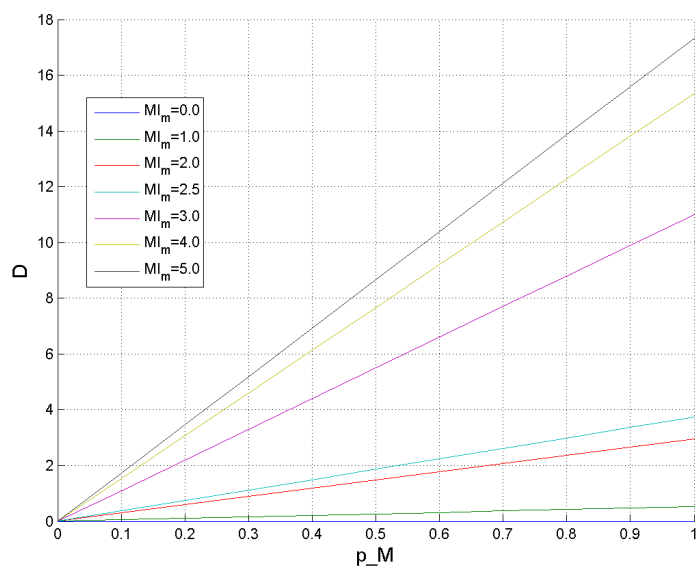

(c) Dependency on $p_{M}$ Fig. 9. Average distance of user movement $\bar{d}$ to achieve best $M I \geq M I_{\text {thresh }}$ in UMi scenario without (top) or with relays (bottom)

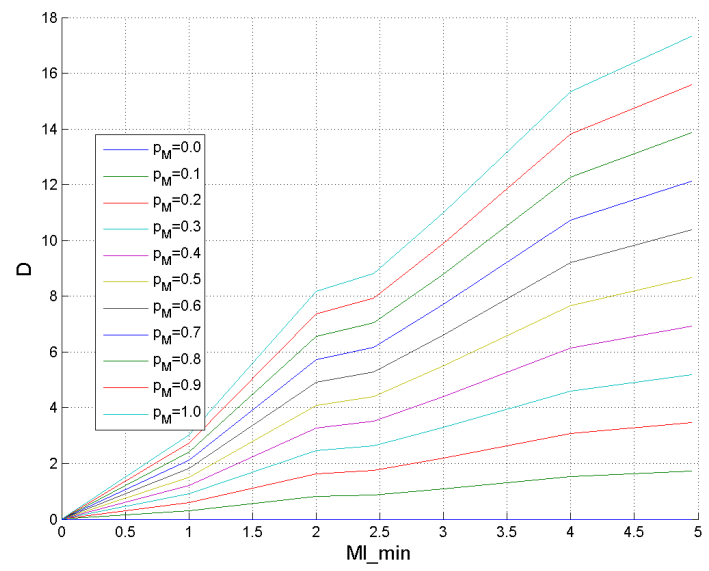

(b) Dependency on $M I_{\text {thresh }}$

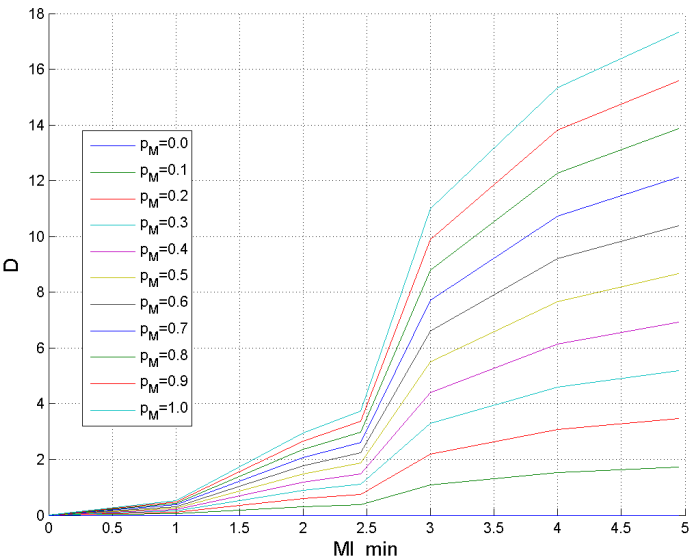

(d) Dependency on $M I_{\text {thresh }}$

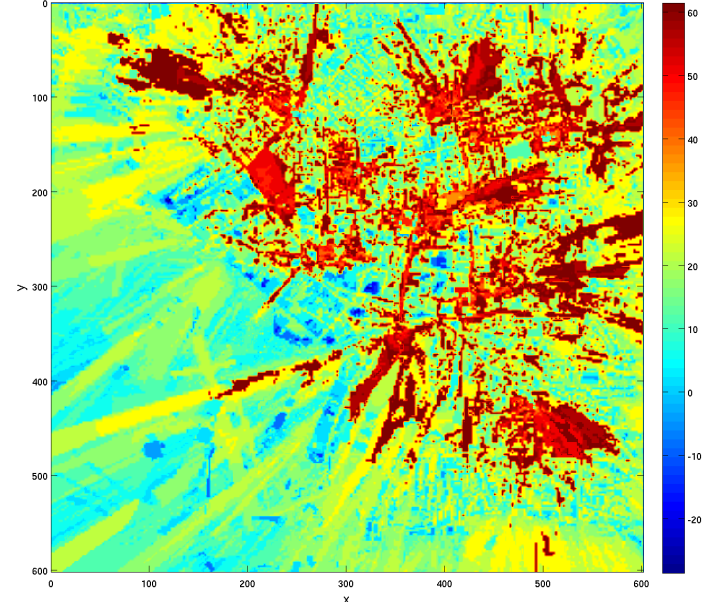

(a) $S I N R / d B$ of region using all 13 relays

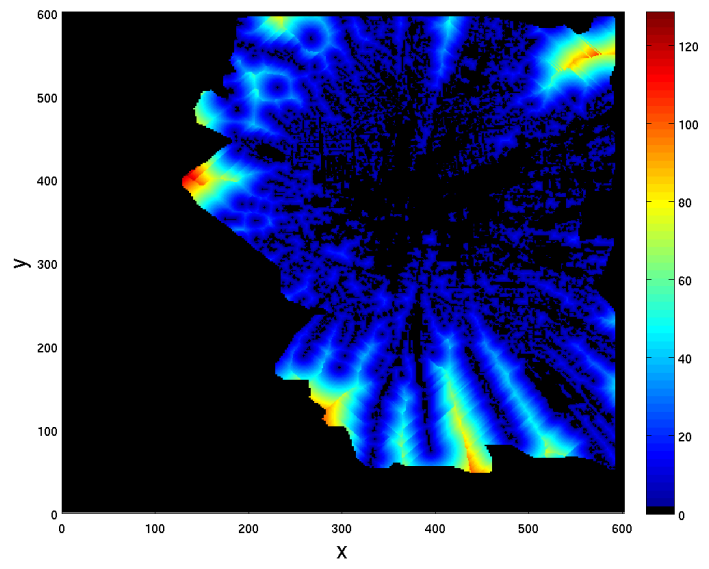

(b) Distance of movement $\bar{d} / m$ to achieve best $M I$

Fig. 10. Jersey scenario results (one step in $\mathrm{x}, \mathrm{y}$ direction is $3.5 \mathrm{~m}$ ). The required movement is only huge at the cell border, as expected. 